\title{
A Simulation Platform Prototype for Evaluating Alternative Scenarios of Members Integration in Virtual Organizations
}

\author{
${ }^{1}$ A. A. Castro, ${ }^{2}$ G. G. Vieira, ${ }^{3}$ M. L. R. Varela, ${ }^{4}$ G. D. Putnik, ${ }^{5}$ J. Machado \\ University of Minho, School of Engineering, \\ ${ }^{1,2,3,4}$ Department of Production and Systems \\ ${ }^{5}$ Department of Mechanical Engineering \\ 1arraiscastro@gmail.com, ${ }^{2}$ gaspar vieira@hotmail.com, \\ ${ }^{3}$ leonildeddps. uminho.pt, ${ }^{4}$ putnikgdedps.uminho.pt, ${ }^{5}$ jma- \\ chado@dem. uminho.pt
}

\begin{abstract}
Companies are facing growing challenges motivated by globalization. A globalized market means that the number of companies with which they will need to compete to maintain or enlarge their market share is increased. Additionally, it also brings greater opportunities to conquer new markets and increase existing market shares, since the geographical, political and economical boundaries are gradually being removed. This paper proposes and illustrates the use of a platform prototype for enabling the evaluation and selection of attractive members for being included as members in a virtual organization. An extended simulation study is described and results obtained presented which show advantage of a used dynamic evaluation and selection model, based on a varying set of criteria.
\end{abstract}

Keywords: Simulation platform, dynamic evaluation model, virtual organization, suppliers and partners selection.

\section{Introduction}

In this paper a multi-agent approach is presented to provide operational support for virtual organizations and collaborative networks, along with a proposed platform prototype that can be applied to multiple business areas, supporting the rapid integration of new businesses and the community of the member with which they operate. The proposed platform is particularly focused on the process of identification, evaluation and selection of suppliers and businesses and integrates services based on the cloud to increase its flexibility and scalability. In order to provide a contribution in this context, besides the existence of some existing work in this area [1-5], our proposal is based on previous contributions, which include [6-11].

Regarding the problem of selection and evaluation of suppliers, as a problem of multi-criteria decision (MCDM), the main contribution of this work was the development of an approach that, jointly, (1) supports, in a complete manner, the process of decision based on multiple criteria in the identification, selection and evaluation of suppliers, (2) using a dynamic model capable of integrating a variable number of criteria

adfa, p. 1, 2011.

(C) Springer-Verlag Berlin Heidelberg 2011 
(spatial variation) and their associated values over time (temporal variation, including past, present and future data), (3) supporting the inaccuracy and lack of confidence, using fuzzy logic for evaluation criteria, allowing (4) applying different weights to different time stages or evaluation criteria, adapted from previous work in [6-11].

This paper aims at analyzing the ability of the proposed platform to identify, evaluate and select a best suited partner and/or supplier, for acting as collaborating member in a VO (Virtual Organization) context, according to its previous performance and present ranking, by using the dynamic approach underlying to this work [6-11]. Therefore, we present the results of a simulation carried out on the implementation of the proposed approach regarding an illustrative example used. First is described the process through which the simulation was performed. Then, the alternative application scenarios are described and finally the obtained results are analyzed and its practical implications explained.

\section{Definition of the proposed platform}

The work performed led to the definition of a proposed platform to support the life cycle of collaborative networks $(\mathrm{CN})$ and virtual organizations (VO), based on a community of software agents [11-18] that cooperate to support the operation of the network. The platform is designed to promote high levels of scalability through its modular approach based on autonomous and encapsulated components, supported by a flexible and moldable infrastructure, based on a distributed approach, which integrates local systems to companies, virtualized systems and cloud-based services. Besides supporting the creation, operation and dissolution of virtual enterprises in the context of collaborative network, the platform is designed to support the rapid identification and selection of partners and suppliers that will consolidate the approach to a business opportunity identified and captured.

The work also resulted in the definition of a dynamic approach capable of supporting decision-making processes that take into account not only the proposals received from a number of suppliers or business partners, but also incorporate information relating to historical performance and its future projection. This dynamic nature of the approach allows reducing the risk inherent in the decision-making process, particularly when various possible solutions must be evaluated in a balanced way in the context of a comprehensive set of evaluation criteria. To enable evaluation of different sets of criteria for past, present and future data, the approach allows to evaluate, in a more balanced way, different providers. By incorporating the treatment of uncertainty, it better supports its use even in scenarios where the completeness and quality of data is not guaranteed.

The research results have an enlarged spectrum of use as it was designed and based on a dynamic multi-criteria decision model (DMCDM) [6-11] for evaluating alternative proposals that are not restricted to a given business model or particular sector. On the other hand, it can be highly beneficial regarding competitiveness of companies where we want to apply it, by allowing the instantiation of collaborative networks that enable 
the creation of synergies and sustain symbiotic relationships in order to expand the potential market, promote a more agile response to market changes, and faster response to changing competition.

The platform proposal supports the creation, operation and dissolution of collaborative networks. One of its main pillars is its ability to decide which are the best partners to establish the network, which are the most suitable companies to integrate VEs (Virtual Enterprises) created on its context and which are the best companies to implement the provision of services or components required to meet the requests about received orders, as illustrated next through Figure 1.

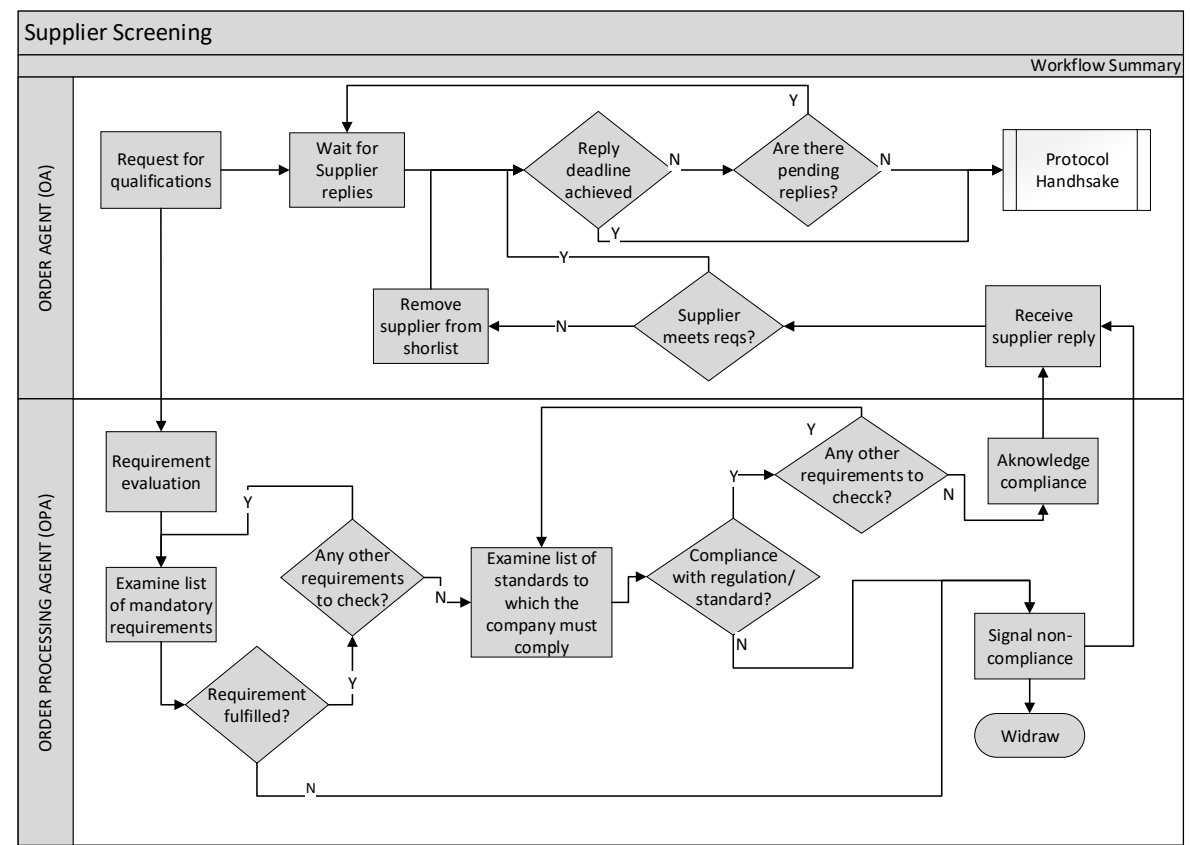

Fig. 1. Business screening workflow.

The businesses evaluation and selection process start with the submission of a request for qualification (RFQ) from a order agent (OA) to the order processing agents (OPAs), according to the agreed protocol, stating which values it wants to receive (for example, price, delivery time and lead time). Additionally, the OA may highlight the evaluation criteria it will use, if appropriate. After receiving an RFQ, each of the OPAs starts a budgeting process. They calculate the price and may also interact with production agents (PA), associated with their Company, in order to obtain the delivery and lead times it can propose, according to the current production schedule.

Additionally, the production agents may report the production capacity during the period, if that parameter is part of the negotiation protocol. Finally, the OPA will issue the proposal to the OA, according to the defined protocol (Figure 1).

The Order Agent which submitted the initial RFQ will evaluate all proposals/ quotes it received, comparing the criteria it established for evaluation. It may include criteria associated with the proposal (price, delivery time, lead time) and criteria associated 
with the business performance (On Time Delivery Performance, Defect Free delivery, Delivery delay mitigation, Defect Mitigation). The evaluation will be performed using historical information, information contained in the received quotes and also forecasting data.

To evaluate the potential associated to our proposed platform we decided to analyze to which extend the underlying dynamic approach really supports the process of evaluation and selection of the best proposals on a given context of application.

Confirmed to obtain good results for some scenarios tested, progress was made towards the creation of an interactive prototype that supports the generation of multiple requests for proposals through successive iterations, submitted to an arbitrary set of businesses. To allow for simulations based on realistic scenarios, the prototype was extended to allow partners (suppliers and/or businesses) consulted to be associated with a matrix of rankings about their profile, in order to introduce a random component, based on known performance of these suppliers/businesses and standards associated to their evolution. This extension of the prototype was used to generate multiple simulations with different configuration parameters for analysis of results and assessment of the dynamic model performance.

To facilitate the execution of multiple analyzes and it's viewing by multiple stakeholders, the prototype was developed using web technologies (PHP, Javascript, CSS, HTML) and on a database MySQL, and focused on the application of the underlying dynamic approach.

The prototype supports the viewing of the main application steps of the dynamic selection and evaluation approach, which in this work was applied to a given scenario of application. Additionally, it allows the execution of various types of simulations, implemented as described next.

To evaluate the performance of the dynamic evaluation approach, it was decided to carry out simulations of its application using the dynamic generation of proposed applications and the evaluation of the answers received. The evaluation was based on three different approaches: (1) a dynamic evaluation, covering the use of historical matrices, present data (resulting from proposals received) and forecasts; (2) assessment regarding historical and present information; (3) evaluation exclusively based on the proposals received.

Table 1. Simulation parameters definition

\begin{tabular}{ccc}
\hline Parameter & Meaning & Value \\
\hline $\mathrm{n}$ & Number of iterations & $10,100,200$ \\
\hline $\mathrm{m}$ & Number of approaches used & 3 \\
\hline $\mathrm{w}$ & Number of suppliers consulted & 6 \\
\hline $\mathrm{h}$ & Number of historical evaluation criteria & 4 \\
\hline $\mathrm{q}$ & Number of present evaluation criteria & 2 \\
\hline $\mathrm{f}$ & Number of future evaluation criteria & 2 \\
\hline $\mathrm{p}$ & Number of proposal requests made on each iteration & $10,20,50$ \\
\hline
\end{tabular}

To analyze the results obtained by applying our underlying dynamic model we proceeded to its application on an illustrative scenario about the selection of a business, among a set of 6 available for the production of a particular product and knowing some 
additional features, which will be described in more detail below, to allow clearly illustrate the potential of the deployed prototype.

The simulation process begins with its preparation. The first set of simulation of the preparation process of the tasks consists on defining the parameters that will condition its implementation. This set includes the number of iterations to be performed, the number of queries to simulate by iteration, the group of suppliers to be consulted, the number of evaluation criteria to be used and the approaches on which they are used. Additionally, matrices are configured for historical data view and associated future data prediction, along with histograms that are used to simulate the behavior of businesses. Table 1 shows the chosen parameters.

Next, it is needed to define the criteria to be used for historical, present and future evaluations. To make the clearest illustration in terms of prototyping, it was selected a representative set of criteria used, related to different kind of cost, as expressed in Table 2 , and this data was jointly used with the information provided through the criteria used for historical and future evaluation of businesses delivery times in order to better support decision making regarding the these underlying criteria.

Table 2. Criteria definition

\begin{tabular}{|c|c|c|}
\hline Moment & Criterion & Description \\
\hline Past & Cost practiced in the past (CPP) & costs charged by the supplier to supply the product in the last 12 months. \\
\hline Past & Total time for delivery (TTD) & $\begin{array}{l}\text { Total time elapsed between the award and the delivery of products from the } \\
\text { award date in the past, for orders of the same product. }\end{array}$ \\
\hline Past & Delivery performance (DPE) & $\begin{array}{l}\text { Previous performance of the supplier in terms of timely delivery of orders } \\
(0-100 \%)\end{array}$ \\
\hline Past & Quality performance (DP) & $\begin{array}{l}\text { Previous performance of the supplier in terms of delivery of orders without } \\
\text { defects }(0-100 \%)\end{array}$ \\
\hline Present & Cost per unit $(\mathrm{CPU})$ & Cost proposed by the supplier for the execution of one unit $(€)$ \\
\hline Present & $\begin{array}{l}\text { Total time for delivery, for pre- } \\
\text { sent (TTD) }\end{array}$ & $\begin{array}{l}\text { Total time elapsed between the award and the delivery of products from } \\
\text { the award of the contract. }\end{array}$ \\
\hline Future & Estimated unit cost (EUC) & $\begin{array}{l}\text { Estimated cost per unit of the same product in the future, according to the } \\
\text { evolution of the in supplier's prices }\end{array}$ \\
\hline Future & $\begin{array}{l}\text { Total time for delivery, for fu- } \\
\text { ture (FTTD) }\end{array}$ & Total delivery time estimated per product unit. \\
\hline
\end{tabular}

For the implementation of the dynamic model, the various evaluation criteria were mapped on their type ("higher is better" or "lower is better"), uncertainty, confidence level. In addition, the $\alpha$ values were defined and $\beta$ for each criterion and $\lambda=1$ [6-11]. The values used as a reference are shown in Table 3.

To evaluate the criteria were used three approaches related to dynamic evaluation model underlying to this work, which were the following: 1. Historical, present and future evaluations (HPF); 2. Historical and present evaluations (HP), and 3. Only present evaluation $(\mathrm{P})$. Therefore, these three approaches require the use of one up to three evaluation matrices, which express the total cost underlying each candidate supplier or business ranking through an underlying data fusion approach used within our dynamic decision model [6-11].

In practice it is considered the same reference time for all repetitions of query request, performing successive simulations of the application of the evaluation approaches at this reference time, and next the results are compared. 
Each of the consulted businesses submits a proposal for providing a unit of product, randomly generating values proposed for the unit cost and for the total time of delivery (corresponding to the sum of the production time and the order preparation time).

Table 3. Criteria parameters associated with the selected criteria

\begin{tabular}{cccccccc}
\hline Moment & Criteria & Type & Uncertainty & Confidence & $\alpha$ & $\beta$ & $\lambda$ \\
\hline Past & CPP & Lower is better & 0 & $100 \%$ & 1 & 0.67 & 1 \\
\hline Past & TTD & Lower is better & 0 & $90 \%$ & 0.8 & 0.67 & 1 \\
\hline Past & DPE & $\begin{array}{c}\text { Higher is bet- } \\
\text { ter }\end{array}$ & $2 \%$ & $80 \%$ & 0.8 & 0.67 & 1 \\
\hline Past & DP & $\begin{array}{c}\text { Higher is bet- } \\
\text { ter }\end{array}$ & $2 \%$ & $80 \%$ & 0.8 & 0.67 & 1 \\
\hline Present & CPU & Lower is better & 0 & $100 \%$ & 1 & 1 & 1 \\
\hline Present & TTD & Lower is better & 0 & $100 \%$ & 0.8 & 0.67 & 1 \\
\hline Future & EUC & Lower is better & $5 \%$ & $80 \%$ & 0.6 & 0.67 & 1 \\
\hline Future & FTTD & Lower is better & $5 \%$ & $60 \%$ & 0.6 & 0.67 & 1 \\
\hline
\end{tabular}

To support the randomness in terms of generation of proposals, each business has been classified according to four main areas: (1) delivery optimization potential, emphasizing the efforts the business has done, namely to reduce the delivery time; (2) potential changes in prices, reflecting the trend observed by the business to increase, decrease or maintain the prices offered; (3) risk of late delivery, reflecting businesses' performance in terms of delivery and the potential of further delays; (4) the risk of defects, reflecting the likelihood of defects in future deliveries.

The classification of the businesses on the areas mentioned allowed introducing random behavior in terms of proposals submitted by them. To generate a new value for each area were used random number between zero and ninety-nine, generated by computer. The random numbers were used as the input key to a histogram that maps each of the areas aforementioned for each business.

The presentation of data is done in order to permit adequate visualization of each type of data analysis and matching results, allowing different views, with more or less detailed information.

Figure 2 below illustrates the prototype's interface regarding the simulation results obtained after 20 iterations, each of which covering the evaluation of 20 proposals about each of the 6 analyzed businesses. For each iteration were added costs generated by each of the approaches according to the data provided by them, taking into account the profile associated with each business concerning the occurrence of faults or delays. In the generated results table presented, we can see the green and underlined values, which correspond to the lower costs (corresponding to the best solution), and in red the highest costs. For example, from a quick look at the data in this figure, it is clear that the dynamic evaluation approach (HPF) enabled to provide the best results in all of the iterations for the scenario under study, by comparison with the other simpler alternative approaches (HP and P).

The prototype allows alternative views of results, along the detailed simulation process. Namely, allowing following the evaluation process of winning proposals for each of the alternative approaches considered, and consultation of the respective total costs. 
The prototype also provides an additional mode of simulation, to facilitate the generation of a large number of records and their subsequent analysis. Supporting the definition of the number of iterations ( $\mathrm{n}$ ) and consultations $(\mathrm{m})$, and the same are associated with the 6 businesses considered in this example.

\section{Protótipo}

Avaliação Dinâmica de Parceiros e Fornecedores

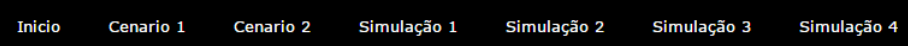

Simulação 3

Parâmetros de execução

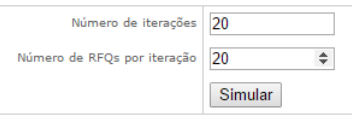

Custos Totais

\begin{tabular}{|c|c|c|}
\hline HPF & HP & $\mathbf{P}$ \\
\hline$\underline{8079990}$ & 8185464 & 8180018 \\
\hline$\underline{8042292}$ & 8185760 & 8152896 \\
\hline 7994366 & 8160721 & 8173538 \\
\hline$\underline{8034780}$ & 8179067 & 8188997 \\
\hline$\underline{8055423}$ & 8173805 & 8189221 \\
\hline$\underline{7983303}$ & 8175857 & 8197531 \\
\hline 7976529 & 8169742 & 8142604 \\
\hline$\underline{8047438}$ & 8180954 & 8205627 \\
\hline$\underline{8047103}$ & 8173552 & 8185954 \\
\hline$\underline{8022291}$ & 8192663 & 8172476 \\
\hline$\underline{8012058}$ & 8207674 & 8195321 \\
\hline$\underline{7999131}$ & 8191165 & 8182396 \\
\hline 7995098 & 8174513 & 8172380 \\
\hline 8067429 & 8192505 & 8178337 \\
\hline$\underline{8023263}$ & 8183827 & 8168594 \\
\hline$\underline{8054579}$ & 8198682 & 8184529 \\
\hline$\underline{8019691}$ & 8177107 & 8207831 \\
\hline$\underline{8045121}$ & 8166652 & 8186767 \\
\hline$\underline{8049537}$ & 8184637 & 8168227 \\
\hline$\underline{8007012}$ & 8170973 & 8204664 \\
\hline
\end{tabular}

Fig. 2. Simulation results visualization.

In this case, the prototype generates $\mathrm{x} * \mathrm{~m} * 6$ random proposals and evaluates them using each of the three approaches considered for this purpose in this study. Once the evaluation, the prototype selects the most advantageous solution, considering the profile of each business (regarding their tendency to deliver orders late or defective), and the resulting costs. At the end we can see a graph comparing the performance of the three approaches used.

The Figure 3 below illustrates the execution of the simulation process for 100 iterations, each of which includes 20 proposal requests (resulting in a total of 2,000 consultations, which generated 12,000 proposals, and these have been evaluated in the context 
of each of the three approaches under consideration, resulting in a total of 36000 ratings). According to defined profiles, the dynamic (HPF) approach was the only one that did enable to generate the best solution in 1071 consultations. Moreover, in 1808 cases it reached the best solution in conjunction with one or both alternative approaches.
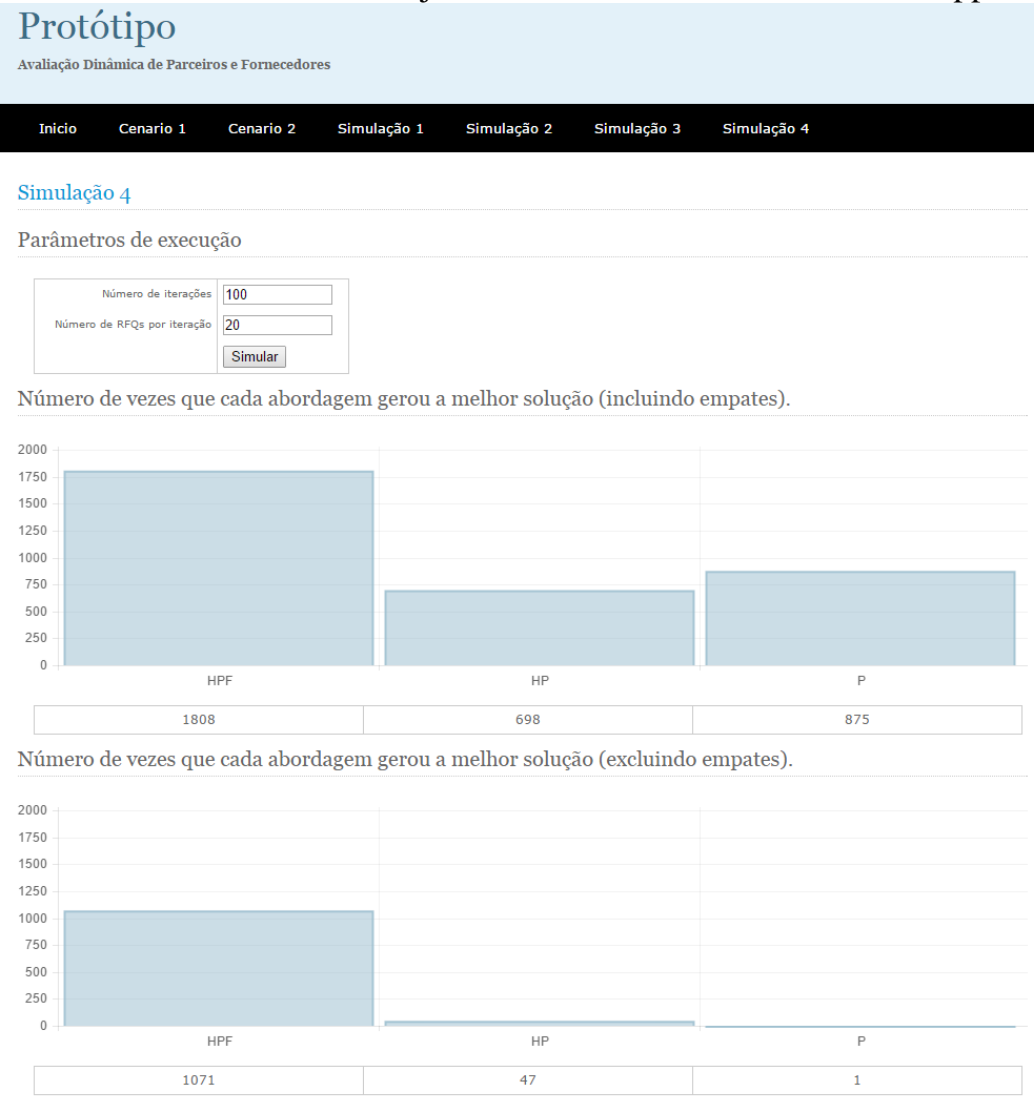

Fig. 3. Visualization of the results of the evaluation process for 2000 consultations and 12,000 proposals analyzed.

The diverse records execution results were recorded in databases, enabling to further make queries and export data and results for further analysis, namely through external tools such as SPSS and Microsoft Excel.

Comparing the cost of each of the solutions generated by each of the three approaches considered we can realize that the dynamic approach was the one that did produce the economically most advantageous solution, as we can see through Figure 4, which expresses the cost of each solution and the cost of the best one.

In practice, since the dynamic approach is responsible for the largest number of best proposals in economic terms, its value is always closest to the best solution value. 


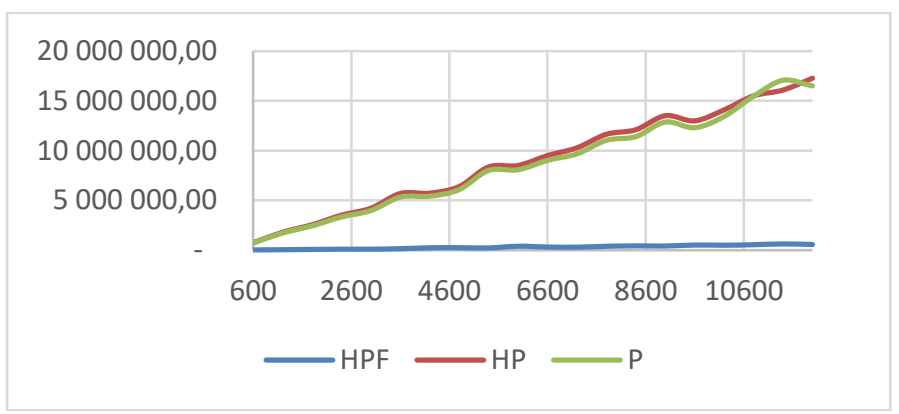

Fig. 4. Evolution of the difference in cost between the three approaches and the best solution obtained on each iteration.

\section{Conclusion}

In a collaborative network, relations between integrating members generate information that is highly valuable to support a useful decision-making process, minimizing the costs associated to its operation and thus contributing to the maximization of potentially associated income opportunities. In order to provide a contribution in this context, besides interesting existing work in this area, such as [1-5], our proposal is based on previous work, such as [6-11]. Moreover, we did not come across any proposal that jointly considers the application of a dynamic multi-criteria decision approach, integrating a data fusion model for improving collaboration and better supporting businesses integration within a virtual enterprise context, not being restricted to a given business model or particular industrial sector. Therefore, in intended to contribute for properly supporting the operation of collaborative networked organizations, and enhancing their continuous reconfiguration process, based on market evolution, Company's members or business partners performance, network size and associated requirements, along with a varying set of parameters for evaluating and selecting appropriate business partners in a dynamic and flexible way. In this study it became clear, through the simulations results obtained, that there are big differences in terms of costs regarding the application of different approaches for alternative businesses' of partners' evaluation and subsequent selection. In all the simulations performed, our proposed dynamic evaluation model, which includes not just present and past data, but also future predictions, produced the best results in the majority of the evaluations carried out. There are several factors that affect the greater or lesser efficiency of each evaluation approach. For the analysis carried out in this work we used a representative set of businesses, through an industrial example. The quality and scope of existing historical data affects the results produced. Moreover, the approaches can be used in environments with more or less amount of uncertainty, and enable the use of distinct sets of parameters (e.g. confidence, accuracy, weights), which enable filtering of uncertainty and producing a final rating according to the importance of the various evaluation criteria used by each Company. 


\section{Acknowledgements}

This work has been supported by COMPETE: POCI-01-0145-FEDER-007043 and FCT Fundação para a Ciência e Tecnologia within the Project Scope: UID/CEC/00319/2013.

\section{$5 \quad$ References}

1. Camarinha-Matos, L., \& Afsarmanesh, H. (2001). Virtual Enterprise Modeling and Support Infrastructures: Applying Multi-agent System Approaches. In Carbonell, J. and Siekmann, J. (Eds.), Multiagents Systems and Applications, pp. 335-364, Sanibel Island, FL, 2001.

2. Ghiassi, M., \& Spera, C. (2003). Defining the Internet-based supply chain system for mass customized markets, Computers \& Industrial Engineering 45, 17-41.

3. Malucelli, A., Rocha, A., \& Oliveira, E. (2004). B2B Transactions enhanced with ontology-based services. ICETE 1, page 10-17. INSTICC Press.

4. Eymann, T., Müller, G., \& Strasser, M. (2006). Self-Organized Scheduling in Hospitals by Connecting Agents and Mobile Devices. In: Kirn, S., et al (Eds.): Multiagent Engineering - Theory and Applications in Enterprises: Int. Handbooks on Information Systems, Springer, Heidelberg 2006, 319-337.

5. Smirnov, A., Sheremetov, L., Chilov, N., Sanchez-Sanchez, C. (2006). Agent-Based Technological Framework for Dynamic Configuration of a Cooperative Supply Chain. Multiagent based Supply Chain Management, Vol. 28, 2006, pp. 217-246.

6. Ribeiro, R. A., Falcão, A., Mora, A., \& Fonseca, J. M. (2013). FIF: A fuzzy information fusion algorithm based on multi-criteria decision-making. Knowledge-Based Systems.

7. Jassbi, J. J., Ribeiro, R. A. \& Varela, L. (2014). Dynamic MCDM with Future Knowledge for Supplier Selection. Journal of Decision Systems, 23 (3), pp. 232-248. ISSN 1246-0125.

8. Campanella, G., \& Ribeiro, R.A. (2011a). A Framework for dynamic multiple criteria decision making. Decision Support Systems, Volume 52, Issue 1, December 2011, pp. 52-60.

9. Campanella, G., Ribeiro, R.A, \& Varela, L.R. (2011b). A Model for B2B Supplier Selection. Advances in Intelligent and Soft Computing, Springer, Warsaw, 107, 221-228, Springer, 2011.

10. Campanella, G., Pereira, A., Ribeiro, R.A., \& Varela, L.R. (2012). Collaborative Dynamic Decision Making: a Case Study from B2B Supplier Selection. In Decision Support Systems. Hernández, J.E., et al. (Eds.), LNBIP, Springer Berlin Heidelberg, vol. 121: 88-102.

11. Ribeiro, R.A. (1996). Fuzzy multiple attribute decision-making: a review and new preference elicitation techniques. Fuzzy sets and systems, 78, 155-181.

12. Rabelo, R., Camarinha-Matos, L. M., \& Vallejos, R. (2000). Agent-based brokerage for virtual enterprise creation in the moulds industry. E-Business and Virtual Enterprises, volume 184 of IFIP Conference Proceedings, page 281-290, Kluwer.

13. Thorat, P., \& Sarje, A. (2011). MobiLim: An Agent Based License Management for Cloud Computing. IC3, Volume 168 of Communications in Computer and Information Science, p.335-346. Springer.

14. Tveit, A. (2001). A Survey of Agent-Oriented Software Engineering. NTNU CSGSC, 4, 367.

15. Wang, M., Wang, H., Vogel, D., Kumar, K., \& Chiu, D. K. W. (2009). Agent-based negotiation and decision making for dynamic supply chain formation. Engineering Applications of Artificial Intelligence, 22, 1046-1055. doi:10.1016/j.engappai.2008.09.001.

16. Wooldridge, M. (1999). Intelligent Agents. In: "Multi-agent Systems - A Modern Approach to Distributed Artificial Intelligence", G. Weiss (ed.), The MIT Press, Cambridge, MA.

17. Wooldridge, M., \& Ciancarini, P. (2001). Agent-Oriented Software Engineering: The State of the Art. In Agent-Oriented Software Engineering (Vol. 1957, pp. 55-82). doi:10.1007/3-540-44564-1_1.

18. Xue, X., Li, X., Shen, Q., \& Wang, Y. (2005). An agent-based framework for supply chain coordination in construction. Automation in Construction, 14, 413-430. doi:10.1016/j.autcon.2004.08.010. 